\title{
An Investigation of Bistatic Calibration Techniques
}

\author{
Christopher J. Bradley, Member, IEEE, Peter J. Collins, Senior Member, IEEE, \\ Joaquim Fortuny-Guasch, Member, IEEE, Michael Larkin Hastriter, Senior Member, IEEE, Giuseppe Nesti, \\ Andrew J. Terzuoli, Jr., Member, IEEE, and Kelce S. Wilson
}

\begin{abstract}
Several popular bistatic calibration techniques are investigated and comparisons made between the relative merits of the various techniques. The analysis addresses sensitivity to object alignment error, sensitivity to polarization impurity, and ease of implementation. Both theoretical concepts and practical considerations are discussed based on measurements accomplished at the European Microwave Signature Laboratory of the European Commission Joint Research Center, Ispra, Italy. This facility has the capability to produce far-field fully polarimetric precision bistatic measurements in a 30-cm-diameter quiet zone, suitable for comparing different calibration methods.
\end{abstract}

Index Terms-Bistatic, calibration, measurement, radar.

\section{INTRODUCTION}

$\mathbf{T}$ HE purpose of this paper is to investigate the performance of several existing and proven bistatic calibration techniques. The techniques are evaluated on the basis of several factors, namely: sensitivity of calibration to reference object alignment error, sensitivity to antenna polarization impurity, and ease and efficiency of implementation. The analysis of bistatic radar calibration objects is critical to the measurement process and is provided in our companion paper [1] as a distinct contribution.

The techniques examined in this effort are detailed in [2]-[6]. The difference between these methods lies mainly in their capability to capture the complete distortion characteristics set forth in the distortion error model. These are the major concerns for the accuracy and efficiency of a given calibration technique. Given the wide variance in measurement conditions from one measurement range to another, a particular calibration technique that is optimal for one range may be unreasonable for another. For this reason, calibration techniques that have been used in a wide variety of measurement facilities and scenarios are of interest.

The next section defines essential terms and relationships as well as provides background on the types and applicability of

Manuscript received August 10, 2004; revised December 14, 2004. The views expressed in this paper are those of the authors and do not reflect the official policy of the U.S. Air Force, U.S. Department of Defense, the U.S. Government, or the European Commission. This paper represents the results of research based on information obtained solely from open sources. No agency, whether U.S. Government or otherwise, provided any operational system parameters or performance data in support of the research presented herein.

C. J. Bradley, P. J. Collins, M. L. Hastriter, and A. J. Terzuoli, Jr. are with the Air Force Institute of Technology, Dayton, OH 45433 USA (e-mail: cbradley@ieee.org; peter.collins@ieee.org; michael.hastriter@afit.edu; a.j.terzuoli@ieee.org).

J. Guasch-Fortuny and G. Nesti are with the European Commission Joint Research Center, 21020 Ispra (VA), Italy (e-mail: joaquim.fortuny @ieee.org; giuseppe.nesti@libero.it).

K. S. Wilson was with the Air Force Institute of Technology, Dayton, OH 45433 USA. He is now with Fulbright and Jaworski, Dallas, TX 75201 USA (e-mail: kwilson@ fulbright.com).

Digital Object Identifier 10.1109/TGRS.2005.855130 bistatic calibrations. Section III reviews the three selected calibration techniques that will be evaluated. Sections IV and V discuss the results of the measurements and compares the calibration methods, drawing conclusions on the performance of each.

\section{BACKGROUND}

\section{A. Perspective}

Focusing on its impact on calibration, our companion paper [1] refers to the measured scattering matrix $\mathbf{M}$ given by

$$
\mathbf{M}=\mathbf{R S T}=\left[\begin{array}{ll}
R_{\mathrm{HH}} & R_{\mathrm{VH}} \\
R_{\mathrm{HV}} & R_{\mathrm{VV}}
\end{array}\right]\left[\begin{array}{ll}
S_{\mathrm{HH}} & S_{\mathrm{VH}} \\
S_{\mathrm{HV}} & S_{\mathrm{VV}}
\end{array}\right]\left[\begin{array}{ll}
T_{\mathrm{HH}} & T_{\mathrm{VH}} \\
T_{\mathrm{HV}} & T_{\mathrm{VV}}
\end{array}\right]
$$

where $\mathbf{R}$ and $\mathbf{T}$ are the subsystem distortion matrices, and $\mathbf{S}$ is the theoretical scattering matrix [5]. This paper examines their effect on the overall calibration equation.

\section{B. Types and Applicability of Calibration}

Multiple types of calibrations can be used for a given measurement. Selection of the calibration type depends on: 1) the capability of the measurement facility; 2) measurement conditions; and 3) the degree of accuracy required. Radar calibration can be categorized according to three basic types: amplitude and phase (Type-1), simple polarimetric (Type-2), and full polarimetric (Type-3).

1) Amplitude and Phase (Type-1) Calibration: Type-1 calibration applies a complex constant scale factor to each measured scattering matrix component. The scale factor is usually calculated by measuring an object with a known radar cross section (RCS) and phase center, and comparing the ratio of the measured and theoretical responses. Type- 1 calibrations all have a common form. For each polarization channel $(\mathrm{HH}, \mathrm{VH}, \mathrm{HV}$, and $\mathrm{VV}$ ), the calibrated scattering coefficient is

$$
S_{\text {cal }}=\frac{S_{\text {tar }}-S_{\text {tar,bkg }}}{S_{\text {cal_tar }}-S_{\text {cal_tar,bkg }}} S_{\text {cal_tar,theo }}
$$

where $S_{\text {cal }}$ is the calibrated response of the object. $S_{\text {cal_tar }}$ and $S_{\text {tar }}$ are the responses of the calibration object and the object under test, respectively. $S_{\text {cal_tar,bkg }}$ and $S_{\text {tar,bkg denote the cor- }}$ responding background responses. $S_{\text {cal_tar,theo is the theoretical }}$ response of the calibration object.

The advantage of using a Type- 1 calibration technique is simplicity. Only one calibration reference object is necessary, and validating computations are easy to implement. There are several disadvantages of using only a Type- 1 technique. The key disadvantage is that the calibration object must have a high RCS for every polarization so that the calibration is valid for the complete scattering matrix. As a result, Type-1 techniques are often only used to calibrate copolar channels. In 
addition, Type-1 techniques do not account for distortion in the transmitter and receiver subsystems.

2) Simple Polarimetric (Type-2) Calibration: Polarimetric calibration technique are often used to account for system distortion. The Type- 2 calibration technique, or simple polarimetric calibration, accounts for zero-order antenna polarization distortion effects. Zero-order effects are defined here as polarization distortion due the difference between the two-way transmit/receive gain between each polarization channel. Each of the copolar subsystem distortion terms $R_{\mathrm{HH}}, T_{\mathrm{HH}}, R_{\mathrm{VV}}$, $T_{\mathrm{VV}}$ cannot be determined distinctly, but can be found as products of the receiver subsystem distortion $R$ and transmitter subsystem distortion $T$ for each polarization channel combination. The distortion terms are expressed in a $4 \times 4$ complete system distortion matrix $\mathbf{C}$, which carries the same information as the $2 \times 2$ matrices $\mathbf{R}$ and $\mathbf{T}$. The $\mathbf{C}$ matrix has the form [7]

$$
\begin{aligned}
\mathbf{C} & \equiv\left[\begin{array}{l}
R_{\mathrm{HH}} \\
R_{\mathrm{HV}} \\
R_{\mathrm{VH}} \\
R_{\mathrm{VV}}
\end{array}\right]\left[\begin{array}{llll}
T_{\mathrm{HH}} & T_{\mathrm{HV}} & T_{\mathrm{VH}} & T_{\mathrm{VV}}
\end{array}\right] \\
& =\left[\begin{array}{llll}
R_{\mathrm{HH}} T_{\mathrm{HH}} & R_{\mathrm{HH}} T_{\mathrm{HV}} & R_{\mathrm{HH}} T_{\mathrm{VH}} & R_{\mathrm{HH}} T_{\mathrm{VV}} \\
R_{\mathrm{HV}} T_{\mathrm{HH}} & R_{\mathrm{HV}} T_{\mathrm{HV}} & R_{\mathrm{HV}} T_{\mathrm{VH}} & R_{\mathrm{HV}} T_{\mathrm{VV}} \\
R_{\mathrm{VH}} T_{\mathrm{HH}} & R_{\mathrm{VH}} T_{\mathrm{HV}} & R_{\mathrm{VH}} T_{\mathrm{VH}} & R_{\mathrm{VH}} T_{\mathrm{VV}} \\
R_{\mathrm{VV}} T_{\mathrm{HH}} & R_{\mathrm{VV}} T_{\mathrm{HV}} & R_{\mathrm{VV}} T_{\mathrm{VH}} & R_{\mathrm{VV}} T_{\mathrm{VV}}
\end{array}\right]
\end{aligned}
$$

where the elements $R_{i j}$ and $T_{i j}$ correspond directly to the elements of the $\mathbf{R}$ and $\mathbf{T}$ matrices mentioned earlier. For a simple polarimetric calibration, only the elements lying on the diagonal of $\mathbf{C}$ are calculated. This is analogous to the products of the elements lying on the diagonals of $\mathbf{R}$ and $\mathbf{T}$. All other terms correspond to cross-polarization impurity of the subsystems, the calculation of which requires a full-polarimetric calibration (Type-3). Even though a Type-2 calibration solves for the products of the $R$ and $T$ coefficients, this calibration can give no information about $\mathbf{R}$ and $\mathbf{T}$ separately; the combined matrix $\mathbf{C}$ must be used instead. Though a single object can be used, the Type- 2 calibration requires a minimum of two independent calibration measurements: a single reference calibration measurement, for which the theoretical solution is precisely known; and an additional depolarizing measurement for cross-polarization correction, also with a known scattering matrix.

3) Full-Polarimetric (Type-3) Calibration: Full-polarimetric calibration is able to solve for all eight coefficients of the $\mathbf{R}$ and $\mathbf{T}$ matrices, and thus all 16 terms of the $\mathbf{C}$ matrix. The zero and first-order subsystem distortion effects are all accounted for using Type-3 calibration. First-order effects include the cross-polarization impurity of the individual transmit and receive antennas due to imperfect rotational alignment of the antenna along the radial axis, as well as cross-polarization contamination inherent in the antenna itself.

The Type- 3 technique is the best available for accuracy in calibration at the cost of complexity. A fully polarimetric technique requires three calibration objects whose theoretical scattering matrices are precisely known [6]. A fully polarimetric technique must solve for the coefficients of the distortion matrices separately, as opposed to a Type-2 technique. This neglects the dependent nature of each distortion term on another. A rigorous mathematical process is necessary in order to solve for the subsystem distortion matrices simultaneously. Once the distortion

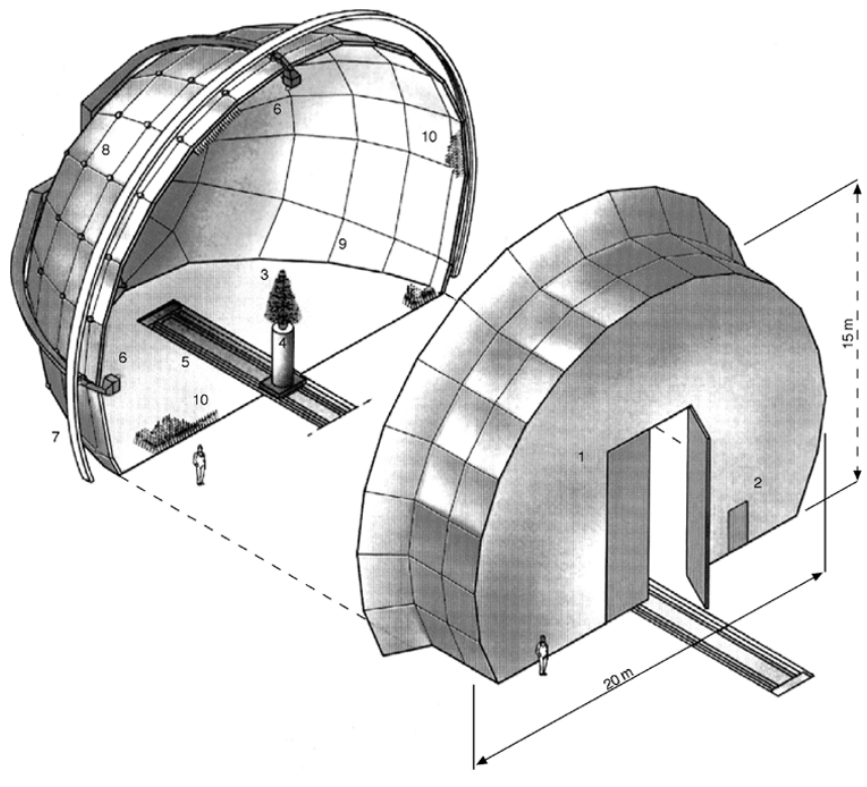

Fig. 1. Exploded view of the EMSL.

matrices are computed, the corrected scattering matrix for the object under test is given by

$$
\mathbf{S}=\mathbf{R}^{-1} \mathbf{M} \mathbf{T}^{-1}
$$

where $\mathbf{S}$ is the corrected scattering matrix, and $\mathbf{M}$ is the measured matrix, with background subtraction performed. This relationship stems from (1), assuming that the subsystem distortion matrices are invertible [5].

\section{Measurement Environment}

All the measurements for this experiment were conducted in the European Microwave Signature Laboratory (EMSL) of the European Commission's Joint Research Centre, Ispra, Italy. This laboratory provides a precise, versatile, and fully polarimetric environment necessary for the measurements. Fig. 1 provides an exploded view of the facility. The horn antennae on the radar sleds at "6" track on a vertical axis around the dome and are located $10 \mathrm{~m}$ from the object positioner at " 4 ." Operating frequencies range from $0.5-26.5 \mathrm{GHz}$. In the range of frequencies used for this experiment $(6-14 \mathrm{GHz})$, this geometry provides a quiet zone of approximately $30 \mathrm{~cm}$ in diameter. ${ }^{1}$

\section{Bistatic CAlibration Methods}

Three existing calibration methods were examined, representing each of the calibration types previously discussed. The following paragraphs briefly describe each method.

\section{A. Basic Type-1 Calibration}

In Type-1 calibration, an object is selected which has a negligible cross-polar return (a sphere or circular disk, for example). The calibrated measurement values for each copolarization $(\mathrm{HH}$ and VV) are computed from (2). Note a different calibration object with a strong cross-polarization response would be required to calibrate for $\mathrm{VH}$ and $\mathrm{HV}$.

\footnotetext{
${ }^{1}$ http://www.jrc.cec.eu.int
} 


\section{B. EMSL Simple Polarimetric Calibration}

The EMSL currently uses the Type-2 calibration method [7]. A circular disk is used for one of the reference objects. This object provides a high specular RCS, a well-defined phase center, and a sufficiently accurate theoretical prediction using physical optics (PO). However, the disk is somewhat difficult to align in the quasi-monostatic (small bistatic angle) configuration. The reference object is used to solve for the $(1,1)$ and $(4,4)$ elements of the $\mathrm{C}$ matrix, i.e., the coefficients $R_{\mathrm{HH}} T_{\mathrm{HH}}$ and $R_{\mathrm{VV}} T_{\mathrm{VV}}$. The $(2,2)$ and $(3,3)$ elements of the matrix are then computed by correcting the amplitudes of the depolarizing object with the $(1,1)$ and $(4,4)$ coefficients already found, and computing the ratio of the measured cross-polar amplitude to the absolute corrected amplitude. The PO approximation for the monostatic scattering matrix of the disk, assuming a perfectly conducting surface is

$$
\mathbf{S}_{\text {disk }}=\frac{2(\pi)^{3 / 2} r^{2}}{\lambda}\left[\begin{array}{ll}
j & 0 \\
0 & j
\end{array}\right]=\left[\begin{array}{cc}
S_{\text {disk }}^{\mathrm{HH}} & 0 \\
0 & S_{\text {disk }}^{\mathrm{VV}}
\end{array}\right]
$$

where $r=$ the radius of the disk in meters, and $\lambda=$ the wavelength in meters. For matrix multiplication by a $4 \times 4$ matrix, the normal $2 \times 2$ scattering matrix format is modified to a $4 \times 1$ vector of the form

$$
\mathbf{S}_{\text {disk }}=\left[\begin{array}{c}
S_{\mathrm{disk}}^{\mathrm{HH}} \\
0 \\
0 \\
S_{\mathrm{disk}}^{\mathrm{VV}}
\end{array}\right]
$$

The elements $C^{1,1}$ and $C^{4,4}$ are solved by relating the measured scattering matrix of the disk $\left(M_{\text {disk }}\right)$ to the theoretical prediction given by

$$
\begin{aligned}
& M_{\text {disk }}^{\mathrm{HH}}=C^{1,1} S_{\text {disk }}^{\mathrm{HH}}+C^{1,2} S_{\text {disk }}^{\mathrm{VH}}+C^{1,3} S_{\text {disk }}^{\mathrm{HV}}+C^{1,4} S_{\text {disk }}^{\mathrm{VV}} \\
& M_{\text {disk }}^{\mathrm{VV}}=C^{4,1} S_{\text {disk }}^{\mathrm{HH}}+C^{4,2} S_{\text {disk }}^{\mathrm{VH}}+C^{4,3} S_{\text {disk }}^{\mathrm{HV}}+C^{4,4} S_{\text {disk }}^{\mathrm{VV}} .
\end{aligned}
$$

For the circular disk, the cross-polar elements are negligible, and we assume that the system crosstalk elements $C^{1,4}$ and $C^{4,1}$ are negligible so the equation simplifies to

$$
\begin{aligned}
& M_{\mathrm{disk}}^{\mathrm{HH}}=C^{1,1} S_{\mathrm{disk}}^{\mathrm{HH}} \\
& M_{\mathrm{disk}}^{\mathrm{VV}}=C^{4,4} S_{\mathrm{disk}}^{\mathrm{VV}}
\end{aligned}
$$

We can now rearrange this equation to solve for $C^{1,1}$ and $C^{4,4}$ and use these coefficients to find the corrected amplitude of the depolarizing object. This allows us to use the wire mesh as a calibration object without knowing its exact RCS. The EMSL uses a mesh of parallel wires as a depolarizer. Two measurements are made on the mesh: one in the vertical orientation and one with the mesh tilted $45^{\circ}$ from vertical. In the vertical orientation, it is reasonable to assume that only the $\mathrm{VV}$ component wave will be reflected, and all others will be transmitted through the mesh, giving the $4 \times 1$ scattering vector the form

$$
\mathbf{S}_{\text {mesh }, \text { vert }}=S_{\text {mesh }}\left[\begin{array}{l}
0 \\
0 \\
0 \\
1
\end{array}\right] \cos ^{2}\left(\frac{\theta}{2}\right)=\left[\begin{array}{c}
0 \\
0 \\
0 \\
S_{\text {mesh,vert }}^{\mathrm{VV}}
\end{array}\right] \text {. }
$$

In the tilted configuration, the vector takes the form

$$
\mathbf{S}_{\text {mesh,tilt }}=\frac{S_{\text {mesh }}}{2}\left[\begin{array}{c}
1 \\
-\cos \left(\frac{\theta}{2}\right) \\
-\cos \left(\frac{\theta}{2}\right) \\
\cos ^{2}\left(\frac{\theta}{2}\right)
\end{array}\right]=\left[\begin{array}{c}
S_{\text {mesh,tilt }}^{\mathrm{HH}} \\
S_{\text {mesh,tilt }}^{\mathrm{VH}} \\
S_{\text {mesh,tilt }}^{\mathrm{HV}} \\
S_{\text {mesh,tilt }}^{\mathrm{VV}}
\end{array}\right]
$$

where $\theta=$ the bistatic angle. This approximation is valid for small bistatic angles. In the tilted orientation, the amplitude of the monostatic $(\theta=0)$ RCS of the mesh should be the same for all polarizations.

To find the corrected values of the mesh in the vertical orientation, we divide the VV-component of the measurement, i.e., the only nonzero component, by $C^{4,4}$, the element of $\mathbf{C}$ corresponding to VV-polarization, i.e.,

$$
S_{\text {mesh,vert }}^{\mathrm{VV}}=\frac{M_{\mathrm{mesh}, \mathrm{vert}}^{\mathrm{VV}}}{C^{4,4}} .
$$

From the corrected magnitude of $S_{\text {mesh,vert, we can find }}$ $S_{\text {mesh }}$, the corrected monostatic scattering coefficient of the vertical mesh from

$$
S_{\mathrm{mesh}}=\frac{S_{\mathrm{mesh}, \mathrm{vert}}^{\mathrm{VV}}}{\cos ^{2}\left(\frac{\theta}{2}\right)} .
$$

Substituting (14) into (12), and selecting the HV and VH polarizations, we get

$$
\begin{aligned}
S_{\text {mesh,tilt }}^{\mathrm{VH}} & =-\frac{S_{\text {mesh,vert }}^{\mathrm{VV}}}{2 \cos \left(\frac{\theta}{2}\right)} \\
S_{\text {mesh,tilt }}^{\mathrm{VH}} & =S_{\text {mesh,tilt }}^{\mathrm{HV}}
\end{aligned}
$$

This is all the information we need to solve for the remaining coefficients of the diagonal $\mathbf{C}$ matrix, i.e.,

$$
\begin{aligned}
C^{2,2} & =\frac{M_{\text {mesh,tilt }}^{\mathrm{VH}}}{S_{\text {mesh,tilt }}^{\mathrm{VH}}} \\
C^{3,3} & =\frac{M_{\text {mesh,tilt }}^{\mathrm{HV}}}{S_{\text {mesh,tilt }}^{\mathrm{HV}}} .
\end{aligned}
$$

After the four calibration coefficients have been computed, the calibration can be performed for a single frequency by the simple matrix multiplication

$$
\mathbf{S}=\mathrm{C}^{-1}(\mathrm{M}-\mathrm{B})
$$

where $\mathbf{S}$ is the calibrated scattering matrix, $\mathbf{M}$ is the measured scattering matrix, and $\mathbf{B}$ is the scattering matrix of the background.

\section{Generalized Dual-Antenna Calibration}

This technique, proposed in [6] and expanded in [5], is the most general technique addressed in current literature. An advantage is that it makes few assumptions about the form of the scattering matrix of each object. It requires that one of the scattering matrices must be invertible and assumes that the scattering matrices are all symmetric. That is, it assumes that crosspolar components are equal. This is a valid assumption for most 
passive reflectors in the monostatic or quasi-monostatic configurations, but is not a valid assumption for bistatic measurements in general. The dependence on the assumption essentially limits the use of this technique to dual-antenna systems calibrated in the quasi-monostatic configuration.

The main limitation of the technique, however, is the requirement that the theoretical scattering matrix of each of the three reference objects be exactly known. In addition, the technique is more mathematically complex than other calibration techniques. Luckily, creation of the mathematical routine is a onetime cost. For a detailed description of this technique, the reader may review the paper by Whitt et al. [6].

\section{RESULTS}

\section{A. Calibration Object Selection}

A set of calibration objects was chosen based on their use in bistatic ranges of practical interest, and additional noncanonical objects were chosen for comparison. In general, the bistatic measurements were performed at a relatively small bistatic angle, assuming that for some objects, the ease and efficiency of monostatic calibration could be extended to small angle bistatic calibration. Measurements were performed for each of the two Tx/Rx combinations. A detailed evaluation of each object is provided in [1].

\section{B. Basic Type-1 Calibration Technique}

This simple technique is presented mainly as a reference for the calibration methods under comparison. An error analysis on the Type- 1 technique is another term for an error analysis of the calibration object prediction itself, which one must determine in order to separate the shortcomings of the calibration from the shortcomings of the object prediction. For the analysis here and in the following sections, the test object for calibration is a metallic disk with a bistatic angle, or antenna separation, of $5^{\circ}$. The frequencies used in the measurements range from 6-14 GHz. The error analysis statistics (Table I) for the Type-1 technique are calculated only on the highest $1-\mathrm{GHz}$ band of the measurement bandwidth, 13 to $14 \mathrm{GHz}$, in order to highlight the effect of misalignment at higher frequencies. The resulting sample populations consist of individual frequency samples.

Upon implementing a Type-1 calibration with the small disk, the statistics of Table I result. The cross-polar levels are indistinguishable from the noise because the cross-polar RCS of the disk prediction was approximated as zero. The disk misalignment was generated normal to the bistatic plane by first precisely aligning the disk with a laser, then using the measurement range's azimuth positioner to create the alignment error.

In order to obtain accurate values for the calibrated crosspolar RCS, an object with high RCS for all four polarization channels must be used. A dihedral tilted $22.5^{\circ}$ from a vertical orientation qualifies [1].

\section{EMSL Simple Polarimetric Calibration Technique}

The first object set used for the analysis of this calibration is the typical set used in the EMSL procedure. For the copolar amplitude reference object (Object 1), the small disk is used.
TABLE I

Average ERror AND $\sigma^{2}$ FOR TYPE-1 CALIBRATION (SMALl Disk)

\begin{tabular}{c|c|c|c|c}
\hline Object Misalignment & VV-error & HH-error & $\underline{\text { VH level }}$ & $\underline{\text { HV level }}$ \\
\hline $0^{\circ}$ & $0.17 \pm 0.01 \mathrm{~dB}$ & $0.10 \pm 0.01 \mathrm{~dB}$ & $-\infty$ & $-\infty$ \\
\hline$l^{\circ}$ & $0.32 \pm 0.04 \mathrm{~dB}$ & $0.35 \pm 0.01 \mathrm{~dB}$ & $-\infty$ & $-\infty$ \\
\hline $2^{\circ}$ & $1.24 \pm 0.04 \mathrm{~dB}$ & $1.28 \pm 0.01 \mathrm{~dB}$ & $-\infty$ & $-\infty$ \\
\hline
\end{tabular}

TABLE II

Average ERror AND $\sigma^{2}$ FOR EMSL CALIBRATION (T1: SMALL DisK, T2: Vertical Wire Mesh, T3: TILTED Wire Mesh)

\begin{tabular}{l|c|c|c|c}
\hline Object Misalignment & VV-error & HH-error & $\underline{\text { VH level }}$ & HV level \\
\hline$T I=0^{\circ}, T 2=0^{\circ}, T 3=0^{\circ}$ & $0.17 \pm 0.01 \mathrm{~dB}$ & $0.10 \pm 0.01 \mathrm{~dB}$ & $-42.0 \mathrm{dBsm}$ & $-42.0 \mathrm{dBsm}$ \\
\hline$T I=1^{\circ}, T 2=1^{\circ}, T 3=1^{\circ}$ & $0.32 \pm 0.04 \mathrm{~dB}$ & $0.35 \pm 0.01 \mathrm{~dB}$ & $-41.4 \mathrm{dBsm}$ & $-41.4 \mathrm{dBsm}$ \\
\hline$T 1=2^{\circ}, T 2=0^{\circ}, T 3=0^{\circ}$ & $1.24 \pm 0.04 \mathrm{~dB}$ & $1.28 \pm 0.01 \mathrm{~dB}$ & $-40.8 \mathrm{dBsm}$ & $-40.8 \mathrm{dBsm}$ \\
\hline$T I=2^{\circ}, T 2=2^{\circ}, T 3=2^{\circ}$ & $1.24 \pm 0.04 \mathrm{~dB}$ & $1.28 \pm 0.01 \mathrm{~dB}$ & $-40.1 \mathrm{dBsm}$ & $-40.2 \mathrm{dBsm}$ \\
\hline
\end{tabular}

For the cross-polar reference objects (Objects 2 and 3, respectively), the wire mesh is used in the vertical orientation, and subsequently with the wires aligned $45^{\circ}$ counterclockwise from vertical. Table II displays the results of the calibration error analysis for this object set.

For the perfect alignment of the calibration objects, the simple polarimetric calibration offers a cross-polarization isolation $(-42 \mathrm{dBsm})$ of about $3 \mathrm{~dB}$ more than the Type-1 calibration using the dihedral tilted at $22.5^{\circ}$. This can be compared with a copolarizing object such as the small disk, which yields a cross-polar RCS of $-39 \mathrm{dBsm}$. The misalignment of the small disk (the most alignment-sensitive of the objects used in this measurement) was the single significant contributor to the overall measurement error.

For the Type- 2 calibration as performed in the EMSL, the object set used in Table II is an appropriate set that can be used. The wire mesh is a known object to return high cross-polar RCS that is strongly correlated with the copolar RCS at a bistatic angle of more than $2^{\circ}$. The relationship can be approximated by cosine squared. The small disk, though very sensitive to alignment, returns the largest bistatic copolar RCS of any object, which enhances the signal-to-noise ratio in the calibration. The EMSL technique could be modified, however, to use the tilted dihedral as a cross-polarization reference object, if the calibration routine is adjusted to use a method of moments (MoM) prediction rather than an approximate prediction based on the vertical wire mesh. This would eliminate one measurement in the calibration - only the small disk and the tilted dihedral, in a single orientation, would need to be used.

\section{Generalized Dual-Antenna Calibration Technique}

This technique, abbreviated as the generalized calibration technique (GCT) in [6], was expected to yield the best results for ideal alignment conditions compared to accurate theoretical solutions. The effect of misalignment of one or more of the reference objects, however, was unknown. Fig. 2 displays the copolar error in a measurement of the metallic sphere calibrated with the GCT, using what is considered to be a nearly optimal object set-the (already defined) three objects used were the small disk (Object 1), the vertical dihedral (Object 2), and the 


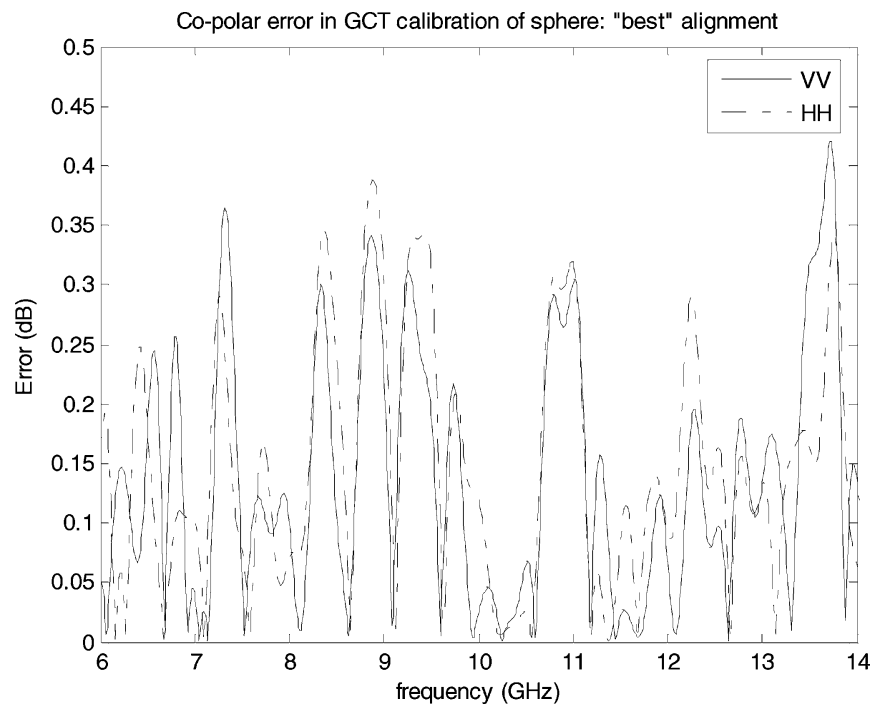

Fig. 2. Error in GCT calibration using small disk, vertical, and tilted dihedral.

dihedral tilted at $22.5^{\circ}$ from vertical (Object 3 ). The normalized scattering matrices for each of these objects have the form

$$
\begin{aligned}
& \mathbf{T}_{\mathbf{1}}=\left[\begin{array}{ll}
1 & 0 \\
0 & 1
\end{array}\right] \\
& \mathbf{T}_{\mathbf{2}}=\left[\begin{array}{cc}
-1 & 0 \\
0 & 1
\end{array}\right] \\
& \mathbf{T}_{\mathbf{3}}=\left[\begin{array}{cc}
-1 & -1 \\
-1 & 1
\end{array}\right] .
\end{aligned}
$$

Every scattering matrix meets the requirement of mutual linear independence.

When misalignment is introduced into the calibration, the calibrated measurement will tend to overestimate the true prediction, and the overestimation will increase with frequency. This is shown in Fig. 3. Table III displays the mean and variance of the RCS error between the calibrated measurements and the prediction of the sphere. As mentioned previously, the statistics are only calculated on the highest $1-\mathrm{GHz}$ band of the measurement, 13 to $14 \mathrm{GHz}$, in order to highlight the effect of misalignment on higher frequencies. As in the simple polarimetric calibration, the misalignment of the small disk was the single significant contributor to the overall measurement error.

The cross-polarization isolation for this case is about $7 \mathrm{~dB}$ higher than the simple polarimetric calibration (Table II). Realizing this, as well as observing from Table III that the penalty resulting from the misalignment of $\mathbf{T}_{\mathbf{1}}$ is considerably more than the penalty of the misalignment of $\mathbf{T}_{\mathbf{2}}$, it is apparent that while the objects' sets are identical, the order in which the objects are implemented in the calibration is important. Only Object 1 is used in the absolute amplitude calibration in the GCT method. Therefore, for any given object set, the most accurate calibration will be that which uses the object with the most precise theoretical prediction, and uses it as Object 1 . In order to optimize the cross-polar isolation in the measurement, one must determine which of Objects 2 and 3 have the most precise prediction, and which of the available equations derived in [6] should be used in the calibration routine to offer the best cross-polarization isolation.

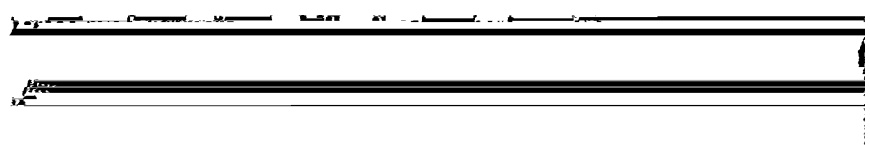

Fig. 3. Error in GCT calibration with $2^{\circ}$ misalignment associated with each reference object.

TABLE III

AVERAGE ERRor AND $\sigma^{2}$ FOR GCT CALIBRATION (T1: SMALl DisK, T2: VERTICAL DiHEDRAL, T3: TILTED DiHEDRAL)

\begin{tabular}{l|c|c|c|c}
\hline Object Misalignment & VV-error & HH-error & $\underline{\text { VH level }}$ & $\underline{\text { HV level }}$ \\
\hline$T 1=0^{\circ}, T 2=0^{\circ}, T 3=0^{\circ}$ & $0.17 \pm 0.01 \mathrm{~dB}$ & $0.10 \pm 0.03 \mathrm{~dB}$ & $-49.2 \mathrm{dBsm}$ & $-51.5 \mathrm{dBsm}$ \\
\hline$T 1=I^{\circ}, T 2=I^{\circ}, T 3=I^{\circ}$ & $0.32 \pm 0.04 \mathrm{~dB}$ & $0.35 \pm 0.01 \mathrm{~dB}$ & $-48.8 \mathrm{dBsm}$ & $-51.5 \mathrm{dBsm}$ \\
\hline$T 1=2^{\circ}, T 2=0^{\circ}, T 3=0^{\circ}$ & $1.24 \pm 0.04 \mathrm{~dB}$ & $1.28 \pm 0.01 \mathrm{~dB}$ & $-47.8 \mathrm{dBsm}$ & $-50.6 \mathrm{dBsm}$ \\
\hline$T 1=2^{\circ}, T 2=2^{\circ}, T 3=2^{\circ}$ & $1.24 \pm 0.04 \mathrm{~dB}$ & $1.27 \pm 0.01 \mathrm{~dB}$ & $-47.8 \mathrm{dBsm}$ & $-50.5 \mathrm{dBsm}$ \\
\hline
\end{tabular}

\section{CONCLUSION}

\section{A. Impact of Calibration Object Selection}

It has been shown that the sensitivity of a particular calibration object to alignment error, though significantly affecting the error in the absolute amplitude calibration, has a less pronounced effect in the determination of the associated antenna polarization distortion matrices (PDMs). This has been demonstrated with the wire mesh and dihedral specifically, where a $2^{\circ}$ misalignment in these objects affects a loss in cross-polarization isolation of about $2 \mathrm{~dB}$.

\section{B. Performance of Type-1, Type-2, and Type-3 Calibrations}

For the measurement parameters of the EMSL, including antenna polarization purity, gain, and signal-to-noise ratio, it has been shown that the gain in cross-polarization isolation in using a Type- 3 technique as opposed to a Type- 1 technique is about $10 \mathrm{~dB}(-39 \mathrm{dBsm}$, as cited in Section IV-C, compared with $-49 \mathrm{dBsm}$ for the Type- 3 technique). The absolute error in the copolar channels is nearly identical, provided that the alignment error and signal-to-noise ratio are kept relatively constant. In general, for any type of calibration, our measurements support the assumption the sources of absolute amplitude error and degradation of cross-polarization isolation are independent. Each calibration technique evaluated here follows the general paradigm that two objects with highly independent scattering matrices are necessary to characterize polarization distortion, and a single object with high copolar RCS is necessary to 
accurately determine the amplitude and phase constant applied to these PDMs.

Given any particular calibration object, it is not difficult to predict the effect of misalignment on the overall error of the calibration. There is a $1: 1$ correspondence between the decibel error per degree of misalignment and the average amplitude error of the copolar channels in the calibration.

These results were obtained in a single measurement environment with a high degree of alignment precision, dynamic range, and cross-polarization purity. Given the ideal conditions of this environment, these conclusions can be extended to any bistatic measurement scenario. For a less sophisticated or more specialized measurement facility, the specific results from the EMSL would most likely represent an upper limit to the gain or loss in measurement accuracy that is obtained by choosing a particular calibration method.

\section{ACKNOWLEDGMENT}

The authors gratefully acknowledge the technical advice and assistance of R. Eigel, M. Temple, G. Lewis, B. Weijers, L. Poles, A. Yaghjian, D. Burnside, R. Marhefka, W. Kent, and D. Schleppi.

\section{REFERENCES}

[1] C. J. Bradley, P. J. Collins, J. Fortuny, M. L. Hastriter, G. Nesti, A. J. Terzuoli, Jr., and K. S. Wilson, "An investigation of bistatic calibration objects," IEEE Trans. Geosci. Remote Sens., no. 10, Oct. 2005.

[2] N. T. Alexander, N. C. Currie, and M. T. Tuley, "Calibration of bistatic RCS measurements," in Proc. Antenna Measurement Techniques Association (AMTA) 1995 Symp., Columbus, OH, Nov. 13-17, 1995, pp. $13-17$.

[3] B. D. Jersak, "Bistatic, fully polarimetric radar cross section calibration techniques and measurement error analysis," Ph.D. thesis, Univ. Texas, Arlington, 1993.

[4] D. Kähny, K. Schmitt, and W. Wiesbeck, "Calibration of bistatic polarimetric radar systems," IEEE Trans. Geosci. Remote Sens., vol. 30, no. 5, pp. 847-852, Sep. 1992.

[5] F. T. Ulaby and C. Elachi, Eds., Radar Polarimetry for Geoscience Applications. Norwood, MA: Artech House, 1990.

[6] M. W. Whitt, W. Michael, F. T. Ulaby, P. Polatin, and V. V. Liepa, "A general polarimetric radar calibration technique," IEEE Trans. Antennas Propagat., vol. 39, no. 1, pp. 62-67, Jan. 1991.

[7] H. B. Mortensen and G. Nesti, "Analysis of polarimetric bistatic scattering from a wire mesh," in 3rd Journées Internationales de la Polarimetrie Radar, Nantes, France, Mar. 21-23, 1995.

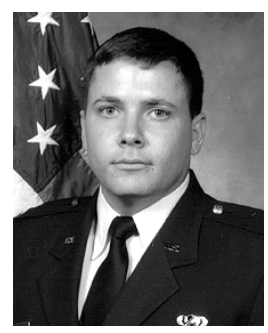

Christopher J. Bradley (M'96) received the B.S.E.E. degree from the Rose-Hulman Institute of Technology, Terre Haute, IN, and the M.S.E.E. degree in the area of bistatic radar polarimetry and computational electromagnetics from the Air Force Institute of Technology, Wright-Patterson AFB, OH, in 1999 and 2001, respectively.

He currently serves the U.S. Air Force as an Engineering Program Manager with current duty as an executive officer in Las Vegas, NV.

Mr. Bradley is a member of Tau Beta Pi and Eta Kappa Nu engineering honor societies.

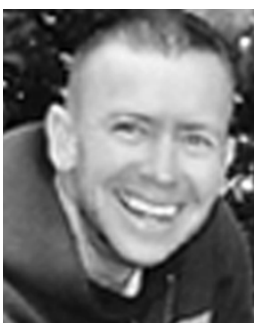

Peter J. Collins (S'84-M'85-SM'00) received the B.S.E.E. degree from the University of Minnesota, St. Paul, and the B.A. degree from Bethel College, St. Paul, in 1985, and the M.S.E.E. and Ph.D. degrees from the Air Force Institute of Technology, WrightPatterson AFB, OH, in 1990 and 1996, respectively.

From 1986 to 1989 , he served a tour of duty at the Air Force Wright Laboratories, Wright-Patterson $\mathrm{AFB}, \mathrm{OH}$, where he performed research in electro-optic component development. In 1990, he served as Engineering Branch Chief with the Radar Target Scatter Division, 6585th Test Group, Holloman AFB, NM. He is currently a Lieutenant Colonel in the U.S. Air Force and Adjunct Associate Professor of electrical engineering at the Air Force Institute of Technology. His research interests are in the area of computational electromagnetics.

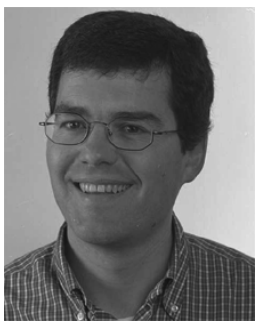

Joaquim Fortuny-Guasch (S'93-A'96-M'04) was born in Tarragona, Spain, in 1964. He received the "Ingeniero" degree in telecommunications engineering from the Polytechnic University of Catalonia, Barcelona, Spain, and the Dr.-Ing. degree in electrical engineering from the Universität Karlsruhe, Karlsruhe, Germany, in 1988 and 2001, respectively.

From 1988 to 1989 , he worked on the design of microwave circuits at Ka-Band in the Electromagnetics and Photonics Engineering Group at UPC. From 1990 to 1992, he was a Research Assistant in the RF Division at the European Space Technology Centre of the European Commission, The Netherlands. Since 1993, he has been with the Directorate General Joint Research Centre of the European Commission, Ispra, Italy. His research interests are in the fields of advanced microwave remote sensing monitoring techniques, calibration techniques for polarimetric radar systems, and the retrieval of biophysical parameters using polarimetric SAR interferometry.

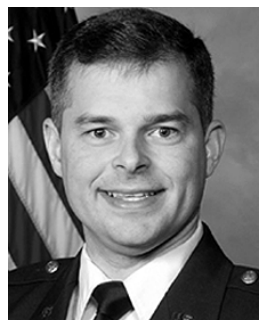

Michael Larkin Hastriter (S'00-M'03-SM'05) received the B.S.E.E. degree from Brigham Young University (BYU), Provo, UT, the M.S.E.E. degree from the Air Force Institute of Technology (AFIT), Wright-Patterson AFB, OH, and the Ph.D. degree in electrical engineering from the University of Illinois, Urbana-Champaign, in 1993, 1997, and 2003 , respectively.

After graduating from BYU, he was commissioned in the U.S. Air Force and is currently on active duty as a Major. After completing his doctoral studies, he was appointed as an Assistant Professor at AFIT teaching in the area of electromagnetics. His research interests include radar cross section prediction, measurement, analysis, and validation as well as scattering centers, bistatic scattering, and periodic corrugated waveguides. He was a distinguished graduate (top 10\%) of AFIT and at Squadron Officer School and has completed in-residence Air Command and Staff College. He has also been named company grade officer of the quarter and field grade officer of the quarter for different organizations. He is an Eagle scout and has served as a troop committee chairman, varsity coach, assistant scoutmaster, and a webelos leader.

Dr. Larkin Hastriter was awarded the Meritorious Service Medal, Air Force Commendation Medal, Air Force Achievement Medal, and the Military Outstanding Volunteer Service Medal for his military service. He is a member of Tau Beta Pi, and Eta Kappa Nu. He was a National Collegiate Engineering Awards winner in 2001. 
Giuseppe Nesti was born in Piteglio, Italy, in 1957. He received the laurea degree in physics from the University of Florence, Florence, Italy, in 1983, with a thesis on remote sensing of natural surfaces by means of microwave radiometry.

In 1985, he joined the European Space Technology Centre, European Space Agency, Noordwijk, The Netherlands, where he was involved mainly in the specification and testing of millimeter-wave radiometers. Since 1988, he has been working at the Joint Research Centre, European Commission, Ispra, Italy, where he has been involved as the key microwave specialist in the design, contruction, testing, and operation of the European Microwave Signature Laboratory (EMSL). He was Responsible of the EMSL operation and scientific program until March 2001. His main research interests concern the experimental investigation of the radar scattering properties of man-made and natural objects, in particular the signal decorrelation induced by natural changes, and the development of calibration procedures for bi- and multistatic polarimetric radar systems.

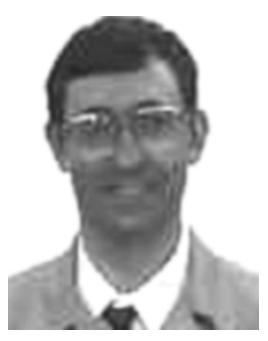

Andrew J. Terzuoli, Jr. (S'77-M'82) received the B.S. degree from the Polytechnic Institute of Brooklyn, New York, the M.S. degree from the Massachusetts Institute of Technology, Cambridge, and the Ph.D. degree from The Ohio State University (OSU), Columbus, in 1969, 1970, and 1982, respectively, all in electrical engineering.

He has been on the civilian faculty of the Air Force Institute of Technology, Wright-Patterson AFB, OH, since late 1982. Prior to this, he was a Research Associate at the ElectroScience Laboratory, OSU, and a member of the technical staff at the Bell Telephone Laboratories in New Jersey. His research areas include computer model-based studies; application of parallel computation, VLSI technology, and RISC architecture to numerical and transform methods in electromagnetics; remote sensing and communication; passive radar; antennas, electromagnetics, wave scattering, radar cross section; machine vision and image processing; and automated object recognition. He has published numerous reports and articles in journals and conference proceedings in these and related areas. His research has been funded by various agencies of the U.S. Government, private industry, and research institutes.

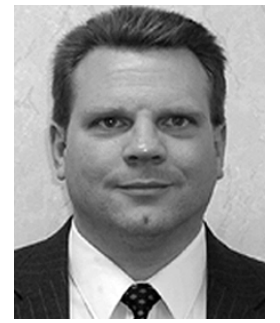

Kelce S. Wilson received the B.S. degree in electrical engineering from the University of Arizona, Tucson, the M.B.A. degree from Chapman University, Orange, CA, the M.S. degree in electrical engineering and the Ph.D. degree in electromagnetic field theory from the Air Force Institute of Technology, Wright-Patterson AFB, OH, and the J.D. degree from Capital University, Columbus, $\mathrm{OH}$, in 1988, 1993, 1994, 1998, and 2005, respectively.

$\mathrm{He}$ is a registered patent agent with the U.S. Patent and Trademark Office, with software security, computational electromagnetics, and radar cross section calculations expertise. $\mathrm{He}$ has spent over 13 years in the Air Force working in both satellite control for the Consolidated Space Operation Center and signature prediction for the Sensors Directorate of the Air Force Research Laboratory. Currently he is an Associate with Fulbright and Jaworski, Dallas, TX. His research has contributed to a better understanding of novel phenomena in radar polarization, processing of digital terrain elevation data, and exploitation of synthetic aperture radar imagery. $\mathrm{He}$ has been an invited speaker in the fields of automatic target recognition and radar noise prediction. His current research area is in antihacking techniques for protecting software from exploitation. 